\title{
Design, Development and Simulations of MHD Equations with its proto type implementations
}

\author{
Rajveer S Yaduvanshi \\ ECE Deptt.AIT, Govt of Delhi, \\ Delhi, India -110031 \\ e-mail-yaduvanshirs@yahoo.co.in
}

\author{
Harish Parthasarathy \\ ECE Deptt. NSIT, Govt of Delhi, \\ Delhi, India-110075 \\ e-mail-harishp@nsit.ac in
}

\begin{abstract}
The equations of motion of conducting fluid in a magnetic field are formulated. These consist of three sets. First is the mass conservation equation, second Navier Stokes equation which is Newton's second law taking into account the force of magnetic field on moving charges. The electrical field effects are neglected as is usually done in MHD. The third set is Maxwell's equation especially to monopole condition along with Ampere's law with the current given by ohm's law in a moving frame (the frame in which the moving particles of fluid is at rest).The mass conservation equation assuming the fluid to be incompressible leads us to express the velocity field as the curl of a velocity vector potential. The curl of the Navier Stokes equation leads to the elimination of pressure, there by leaving with an equation involving only magnetic field and the fluid velocity field. The curl of the Ampere law equation leads us to another equation relating to the magnetic field to the velocity field. A special case is considered in which the only non vanishing components of the fluid are the $x$ and $y$ components and the only non vanishing component of the magnetic field is $z$ component. In this special case the velocity vector potential only has one non zero component and this is known as stream function. The MHD equation in this reduces to three partial differential equations for
\end{abstract} the three functions in $2 \mathrm{D}$ model. $\Psi$ stream function embeds $v_{x}$ and $v_{y}$ components. Application of MHD system prototype has been worked and presented.

Keywords- Lorentz force, Navier Stokes Equation, Maxwell's Equation, Iterative Solution, Prototype.

\section{INTRODUCTION}

Magneto hydrodynamic equation solutions in the field of fluid dynamic have been very recent in many of the applications.. In current era, there has been a growing interest of research in the interaction between ionic currents in electrolyte solutions and magnetic fields. Magneto hydrodynamics (MHD) (magneto fluid dynamics or hydro magnetic) is the academic discipline, which studies the dynamics of electrically conducting fluids. Examples of such fluids include plasmas, liquid metals, and salt water. The word magneto hydrodynamics (MHD) is derived from magnetomeaning magnetic field, and hydro- meaning liquid, and dynamics meaning movement. MHD is the study of flow of electrically conducting liquids in electric and magnetic fields. Magneto hydrodynamics involves then a combination of both electrical and magnetic fields in order to induce mechanical flow in a fluid that is made conductive by dissolving an electrolyte in it. Since the ionic flow in the magnetic field is the cause of the movement of the conductive fluid, then it is necessary to understand the properties of the electromagnetic forces at work.

The idea of MHD [1-5] is that magnetic fields can induce currents in a moving conductive fluid, which create forces on the fluid, and also change the magnetic field itself. The set of equations which describe MHD are a combination of the Navier-Stokes equations of fluid dynamics and Maxwell's equations of electromagnetism

These differential equations have to be solved simultaneously, either analytically or numerically. MHD is a continuum theory and as such it cannot treat kinetic phenomena, i.e. those in which the existence of discrete particles or of a non-thermal velocities distribution are important. The electric currents transmitted in an electrolyte solution interact with the magnetic field to form Lorentz body forces that in turn, drive fluid motion. Lorentz force is the flow in the direction perpendicular to both magnetic and electric fields in conductive, aqueous solutions in the MHD system.

The phenomenon is commonly referred to as magnetohydrodynamics (MHD). The modelling of MHD flow of electrolytes can be often more complex than that of liquid metals since the local electric conductivity is a function of the electrolytes concentration. The MHD flow is governed by classical fluid dynamics and electromagnetic, including a set of coupled partial differential equations that express the conservation of mass continuity and Navier Stokes equation joined to the Maxwell's, current continuity and constitutive equations.

Navier Stokes equation is nonlinear PDE based on Newton's second law. Navier stokes equation dictates velocity not position. When mean free path becomes comparable to the flow characteristics length scale $\mathrm{i}$.e. Molecule length scale that is smaller than mean free path flow deviates from Navier stokes equation. It is quantified by $\mathrm{K}_{n}=\lambda / L$. Solution to Navier Stokes [1-3] equation is called velocity field or flow field description of fluid flow at given point in space and time. Once velocity is solved then flow rate and drag can be evaluated. In the absence of steep gradients in fluid properties NS ceases to be valid and similar when Knudson no increases.

Magnetohydrodynamic (MHD)[3-5] is the theory of the interaction of electrically conducting fluids and electromagnetic fields. In this paper, electrically conducting 
fluid flow constrained in a rectangular MHD duct, where a uniform magnetic field is applied perpendicular to the streamwise direction and to the homogeneous electric field. Our model treats the electrolyte as a conductor, and uses current and momentum conservations. Application to MHD can be to drive and control flows in micro fluidic sensors, networks, astronomy [5-7] and geo-physics and liquid metal cooling of nuclear reactors, electromagnetic casting of metals, MHD power generation and propulsion systems[8-10] .

This document is organized in six main sections. The first section is devoted to the fundamentals of magneto hydrodynamic. In the second section, the magneto hydrodynamic 2D modelling with all relevant formulations are presented. Section three discusses special case for $\mathrm{z}$ axis. Evaluated results are presented in section four. Section five deals with its proto type implementation. Conclusion has been briefed in section six.

\section{MHD FORMULATIONS}

Despite its apparent simplicity, MHD describes a remarkably rich and varied mix of phenomena. MHD theory is a marriage between fluid mechanics and electromagnetism. Subject is one whose development continues to flourish. The MHD flow is governed by classical fluid dynamics and electromagnetic, including a set of coupled partial differential equations.

let there be a conducting fluid having $\sigma$ conductivity, $\rho$ mass density, $p$ Pressure, $v=\eta / \rho$ kinetic viscosity, $\mathrm{R}$ Reynolds Number, $\mathrm{v}$ velocity of fluid and $\mathrm{J}=\rho \mathrm{V}$ mass flux density, $\mathrm{J}=\boldsymbol{\sigma}(\mathrm{E}+\mathrm{V} \times \mathrm{B})$, where $\mathrm{J}=$ Current Density , B is Magnetic Field ,E= Electric Field, F = J X B, where F Lorentz force i.e. force on this current on the fluid per unit volume, Navier Stoke's [1] equation is given by the relation

$$
\rho(v \cdot \nabla v+v, \mathrm{t})=-\nabla \mathrm{p}+\eta \nabla^{2} \mathrm{v}+\mathrm{J} \times \mathrm{B}
$$

\section{Above can be expressed as}

$$
\begin{aligned}
& \rho\left(\Omega \times v+\frac{\nabla^{2} v}{2}+v, \mathrm{t}\right)= \\
& -\nabla \mathrm{p}+\eta \nabla^{2} \mathrm{v}+\mathrm{J} \times \mathrm{B}
\end{aligned}
$$

\section{Where $\Omega=\nabla \times v$ is vorticity.}

$$
\rho\left(\Omega \times v+\nabla^{2} \mathrm{v} / 2+v, \mathrm{t}\right)=
$$$$
-\nabla \mathrm{p}+\eta \nabla^{2} \mathrm{v}+\mathrm{J} \times \mathrm{B}
$$

Taking curl of equation (3) to eliminate pressure term

$$
(\nabla \times(\Omega \times v)+\Omega, \mathrm{t})=v \nabla^{2} \Omega+\nabla \times(\mathrm{J} \times \mathrm{B})
$$

Evolution of 2D solution of the above equation

$$
\begin{aligned}
& \text { As } \vec{v}=\vec{\nabla} \times \vec{\psi} \\
& \Omega=\nabla \times v=\nabla \times \nabla \times \psi \\
& \mathrm{V}=v_{x} \widehat{x}+v_{y} \widehat{y} \quad \text { (for 2D flow) } \\
& \mathrm{B}=B_{z} \widehat{Z}
\end{aligned}
$$$$
\nabla^{2} \Omega=\nabla(\nabla . \Omega)-\nabla \times \nabla \times \Omega
$$$$
\nabla_{\mathrm{x}}(\Omega \times v)=
$$$$
\widehat{k} \quad\left[\frac{\partial}{\partial x}\left(v_{x} \frac{\partial v_{y}}{\partial x}-v_{x} \frac{\partial v_{x}}{\partial y}\right)+\right.
$$$$
\left.\frac{\partial}{\partial y}\left(v_{y} \frac{\partial v_{y}}{\partial x}-v_{y} \frac{\partial v_{x}}{\partial y}\right)\right]
$$$$
\frac{\partial \Omega}{\partial t}=\hat{k} \frac{\partial}{\partial t}\left[\frac{\partial v_{y}}{\partial x}-\frac{\partial v_{x}}{\partial y}\right]
$$

$\nabla \times \nabla \times \Omega=$

$$
\begin{aligned}
& \left.\hat{k}_{[}\left[-\frac{\partial^{3} v_{y}}{\partial x^{3}}+\frac{\partial^{3} v_{x}}{\partial^{2} x \partial y}\right]-\left[\frac{\partial^{3} v_{y}}{\partial^{2} y \partial x}-\frac{\partial^{3} v_{x}}{\partial y^{3}}\right]\right] \\
& \nabla_{\mathrm{X} J \mathrm{~B}}=\hat{k}\left(\frac{\partial v_{x} B_{z}^{2}}{\partial x}-\frac{\partial v_{y} B_{z}^{2}}{\partial y}\right)
\end{aligned}
$$

$\nabla \mathrm{x} \quad \mathrm{B}=(\mu \mathrm{J}+\mu \in \mathrm{E}, \mathrm{t})$

On neglecting displacement current

$\mathrm{J}=\frac{\nabla \times \mathrm{B}}{\mu}$;

We have force per unit volume due to electromagnetic field as

$$
\begin{aligned}
\mathrm{F} & =\mu^{-1}(\nabla \times \mathrm{B}) \times \mathrm{B} \\
& =\mu^{-1} \mathrm{~B} \cdot \nabla \mathrm{B}-\frac{1}{2 \mu}\left(\mathrm{B}^{2}\right)
\end{aligned}
$$

We thus get Navier stroke's equation as

$$
(\nabla \times(\Omega \times v)+\Omega, \mathrm{t})=v \nabla^{2} \Omega+\frac{1}{\mu \mathrm{p}} \nabla \times(\Omega \mathrm{B} \times \mathrm{B})
$$

$$
\begin{aligned}
& \text { Where } \Omega \mathrm{B}=(\nabla \times \mathrm{B})=\mu \mathrm{J} \\
& \text { If } v_{x}=\frac{\partial \psi}{\partial y}, v_{y}=-\frac{\partial \psi}{\partial x}
\end{aligned}
$$

The equation (4) can be reduced in terms of two variable i.e. B Magnetic field and $\psi$ Stream function.

Hence above equation can be reduced 


$$
\begin{aligned}
& \widehat{k}\left[-\frac{\partial^{2} \Psi}{\partial x \partial y} \frac{\partial^{2} \Psi}{\partial x^{2}}-\frac{\partial \Psi}{\partial y} \frac{\partial^{1} \Psi}{\partial x^{s}}-\frac{\partial^{2} \Psi}{\partial y \partial x} \frac{\partial^{2} \Psi}{\partial y^{2}}-\frac{\partial \Psi}{\partial y} \frac{\partial^{\mathrm{s}} \Psi}{\partial^{2} y \partial x}+\right. \\
& \frac{\partial^{2} \Psi}{\partial y \partial x} \frac{\partial^{2} \Psi}{d x^{2}}+\frac{\partial \Psi}{\partial y}\left(\frac{\partial^{\mathrm{s}} \Psi}{\partial y \partial^{2} x}\right)+\frac{\partial^{2} \Psi}{\partial y \partial x} \frac{\partial^{2} \Psi}{\partial y^{2}}+ \\
& \left.\frac{\partial \Psi}{\partial x} \frac{\partial^{\mathrm{I}} \Psi}{\partial y^{\mathrm{s}}}\right]+\widehat{k} \frac{\partial}{\partial t}\left[-\frac{\partial^{2} \Psi}{\partial x^{2}}-\frac{\partial^{2} \Psi}{\partial y^{2}}\right]= \\
& v \widehat{k}\left[-\left[\frac{\partial^{4} \Psi}{\partial x^{4}}+\frac{\partial^{4} \Psi}{\partial x^{2} \partial y^{2}}\right]-\left[-\frac{\partial^{4} \Psi}{\partial y^{2} \partial x^{2}}-\frac{\partial^{4} \Psi}{\partial y^{4}}\right]\right] \\
& +\sigma \hat{k}\left[\frac{\partial}{\partial x}\left[\frac{\partial \Psi}{\partial y} B_{z} 2\right]+\frac{\partial}{\partial y}\left[\frac{\partial \varphi}{\partial x} B_{z} 2\right]\right.
\end{aligned}
$$

As per Maxwell's Ampere's law

$$
\nabla \times \mathrm{E}=-\mathrm{B}, \mathrm{t} \text {; }
$$

Taking curl on both sides and solving

$$
\left(\nabla^{2} \mathrm{~B}-\mu \sigma \mathrm{B}, \mathrm{t}\right)+\mu \sigma \nabla \mathrm{x}(v \mathrm{x} \mathrm{B})=0
$$

When fluid velocity and magnetic field are

Functions of $\mathrm{x}, \mathrm{y}, \mathrm{t}$.

$$
\nabla^{2} B_{0}-\mu \sigma B, \mathrm{t}+\mu \sigma \nabla \times(\mathrm{V} \times B)=0
$$

Here $v_{x}, v_{y}, B_{z}$ are functions of $\mathrm{x}, \mathrm{y}, \mathrm{t}$

$$
\begin{aligned}
& (\nabla \times V \times B)_{x}=(V \times B)_{y_{z} z}-(V \times B)_{z y} \\
& (\nabla \times V \times B)_{y}=(V \times B)_{z x}-(V \times B)_{x_{y} z} \\
& (\nabla \times V \times B)_{z}=(V \times B)_{y, x}-(V \times B)_{x y}
\end{aligned}
$$

Evolution of 2D MHD system can be found under assumed initial and final conditions. Taking initial input as Gaussian pulse $=e^{-(t-t 0) /} \sigma^{2}$

$$
\begin{aligned}
& \left(\nabla^{2} \cdot \mathrm{B}-\mu \sigma \mathrm{B}, \mathrm{t}\right)+\mu \sigma \nabla \times(v \times \mathrm{B})=0 \\
& \left(\nabla^{2} \cdot \mathrm{B}-\mu \sigma \frac{\partial B}{\partial t}\right)+\mu \sigma \nabla \times(v \times \mathrm{B})=0
\end{aligned}
$$

Solving the above equation

$$
\begin{aligned}
& \nabla . \mathrm{B}=0, \\
& \nabla \times \mathrm{E}=-\mathrm{B}, \mathrm{t} \\
& \nabla \times \mathrm{B}=\mu \sigma\left(\mathrm{E}+v_{\mathrm{x}} \mathrm{B}\right) \\
& \nabla \times B=\hat{\imath}\left(\frac{\partial B_{z}}{\partial y}\right)-\hat{\jmath}\left(\frac{\partial B_{z}}{\partial x}\right) \\
& \nabla \times \nabla_{\mathrm{x}} B=\hat{k}\left(-\frac{\partial^{2} B_{z}}{\partial x^{2}}-\frac{\partial^{2} B_{z}}{\partial y^{2}}\right) \\
& \mathrm{V} \times \mathrm{B}=\hat{\imath}\left(\nu_{y} B_{z}\right)-\hat{\jmath}\left(\nu_{x} B_{z}\right)
\end{aligned}
$$

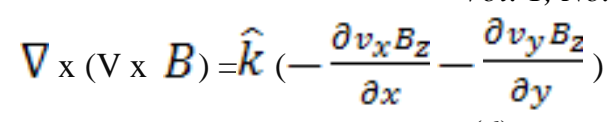

Hence final reduced equation is given below in $\mathrm{B}$ and $\psi$ variables.

$$
\begin{aligned}
& \widehat{k}\left(\frac{\partial^{2} B_{z}}{\partial x^{2}}-\frac{\partial^{2} B_{z}}{\partial y^{2}}\right)-\mu \sigma \widehat{k} \frac{\partial B_{z}}{\partial t}- \\
& \mu \sigma \widehat{k}\left[\frac{\partial}{\partial x}\left(\frac{\partial \varphi}{\partial y} B_{z}\right)-\frac{\partial}{\partial y}\left(\frac{\partial \varphi}{\partial x} B_{z}\right)\right]=0
\end{aligned}
$$

Taking initial magnetic field $B_{z 0}$ as Gaussian and initial electric field negligible. We shall solve this problem for Magnetic field, B and Stream function $\psi$ taking them as function of $\mathrm{x}, \mathrm{y}$ and. From above equation, we observe that velocity of conducting fluid is varying whenever magnetic field is changed and vice versa. Solution of above equation (7) has been worked out by iteration method. As equation (7) has been reduced to only two variables in stream function $\psi$ and magnetic field B. We can solve this equation by Finite Difference Method numerically for computing stream function $\psi$ and their corresponding magnetic field B by iterative solution. Now substituting the value of magnetic field obtained in the problem equation and solving it for new set of stream function $\psi$, we shall continue the same approach till convergence. Here we can assume initial stream function $\psi$ as $2 \mathrm{n} \pi$ radian and then proceed to evaluate their corresponding value magnetic field $\mathrm{B}$. We have also worked this problem with experimental and analytical methods. It has been found[11] from results that computed results are in close proximity. We can get much better results if simulated for $3 \mathrm{D}$ solution but process may be complex.

\section{SPECIAL CASE FOR Z AXIS}

One Special Case for Solution of MHD equation taking only z-axis into account.

As we know from Nervier Stokes equation

$$
\begin{aligned}
& \left(\nabla \times(\vec{\Omega} \times \vec{v})+\frac{\partial \Omega}{\partial t}\right)=v \nabla^{2} \Omega+\frac{1}{\rho} \nabla \times(\vec{J} \times \vec{B}) \\
& \mathrm{J}=\sigma(\quad \vec{v} \times \vec{B}) \\
& v_{x}(\mathrm{t}, \mathrm{x}, \mathrm{y}) \hat{x}+v_{y}(\mathrm{t}, \mathrm{x}, \mathrm{y}) \hat{y} \\
& \Omega_{z} \quad=v_{y x}-v_{x y y} ; \text { angular velocity } \\
& \vec{\Omega} \times \vec{v}=\Omega_{z} \widehat{z} \times v_{x} \hat{x}+v_{y} \hat{y} \\
& =\Omega_{z}\left(v_{x} \hat{y}-v_{y} \hat{x}\right) \\
& \nabla \mathrm{X}(\vec{\Omega} \mathrm{x} \vec{v})=\left[\left(\Omega_{z} v_{x}\right)_{a} x+\left(\Omega_{z} v_{y}\right)_{y}\right]^{\underline{Z}}
\end{aligned}
$$

$$
\nabla \cdot v=0
$$




$$
\begin{aligned}
& v_{x, x}+v_{y y}=0 \\
& v_{x}=\psi, y \\
& v_{y}=-\psi_{\mathrm{a}} x \\
& \nabla \times(\vec{\Omega} \times \vec{v})=\Omega_{z x x} v_{x x}+\Omega_{z y} v_{y} \\
& \Omega_{z}=\psi_{\mathrm{a}} x x-\psi_{\mathrm{a}} y y=-\nabla^{2} \psi \\
& \text { where } \nabla^{2}=\frac{\partial^{2}}{\partial x^{2}}+\frac{\partial^{2}}{\partial y^{2}} \\
& \nabla \times(\vec{\Omega} \times \vec{v})=-\nabla^{2} \psi_{x x}\left(\psi_{y}\right)-\nabla^{2} \psi_{a} y\left(-\psi_{a} x\right) \\
& =\left(\psi_{\mathrm{a}} x \nabla^{2} \psi_{\mathrm{a} y}-\psi_{\mathrm{a}} \nabla^{2} \nabla_{\mathrm{a}} x\right) \\
& =\psi_{\mathrm{a} x} \nabla^{2} \psi_{\mathrm{a} y}-\psi_{\mathrm{a} y} \nabla^{2} \psi_{\mathrm{a} x}-\nabla^{2} \psi_{\mathrm{a} t} \\
& -v \nabla^{2} \nabla^{2} \psi \hat{z}+\frac{\sigma}{\rho} \nabla \times((\vec{v} \times \vec{B}) \times \mathrm{B} \\
& \mathrm{J}=\sigma(\vec{v} \times \vec{B}) \\
& \nabla \cdot \mathrm{B}=0 \quad \text {, } \\
& (\vec{v} \times \vec{B}) \times \vec{B}=\left(\left(v_{x} \hat{x}+v_{y} \hat{y}\right) \times B_{0} \hat{z}\right) \times B_{0} \hat{z} \\
& =(\vec{v} \times \vec{B})-B^{2} \vec{v} \\
& =-B_{0}{ }^{2}\left(v_{x} \hat{x}+v_{y} \hat{y}\right) \\
& =B_{0}{ }^{2}\left(\psi_{\imath} Y \hat{x}-B_{0}{ }^{2} \psi_{x} \hat{y}\right) \text { or } B_{0}{ }^{2} \psi_{z} \hat{y}-B_{0}{ }^{2} \psi_{o} Y \\
& \frac{\sigma}{\rho}\left(\left(B_{0}{ }^{2} \psi_{x}\right)_{a} x+\left(B_{0}{ }^{2} \psi_{y}\right)_{y}\right)=\frac{2 \sigma^{2}}{\rho}\left(B_{0, x} \psi_{x}+\right. \\
& \nabla \mathrm{xB}=\mu \sigma(\vec{v} \mathrm{x} \vec{B}) \\
& \nabla(\nabla \cdot \mathrm{B})-\nabla^{2} \mathrm{~B}=\mu \sigma \nabla \times \quad(\vec{v} \times \vec{B}) \\
& \nabla^{2} B_{0}+\mu \sigma(\vec{v} \times \vec{B})\left(\left(v_{y} B_{0},-v_{x} B_{0}, 0\right)\right.
\end{aligned}
$$

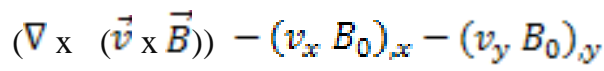

$$
\begin{aligned}
& =-v_{x} B_{0_{x}}-v_{y} B_{0_{y}} \\
& =-\psi_{y} B_{0, x}+\psi_{a x} B_{0 y} \\
& \text { Hence } \nabla^{2} B_{0}-\mu \sigma\left(\psi_{y} B_{0_{x}}+\psi_{x,} B_{0_{y}}\right) \\
& =0
\end{aligned}
$$$$
\hat{y}
$$$$
\left.B_{0 y} \psi_{y}\right)
$$

Here we find solution for $\mathrm{z}$ - axis. We shall assume initial velocity and initial magnetic field by keeping electric field as negligible. We shall compute the changes in velocity and magnetic field iteratively by keeping initial conditions at an assumed value and then evaluate the results which shall follow the MHD principle and plot the simulation results. Basically magnetic and electric field are perpendicular to each other and velocity in the direction of plane is to be seen for $\mathrm{z}$ coordinate.

\section{EVALUATED RESULTS}

Convergence plot for iterative solution of Magnetic field and Stream function have been shown in figure 1- 5. Plot for $\mathrm{B}$, vx and $\mathrm{t}$ has been presented. Velocity profile plots have been shown in fig 1-4. We have obtained two sets of equations after optimization and iterative solution have been worked with numerical technique for convergence using MATLAB. Figure 1- 5 clearly depicts solution to this problem.

We have reduced system to two variables as seen from equation (5 )and (7). For 2D analysis of MHD system figure (1-4) presents magnetic field and velocity profile as function of $\mathrm{x}, \mathrm{y}, \mathrm{t}$. We have observed that velocity variation is dependent on magnetic field and electric field during experimental results We have taken plot for stream function and magnetic field under certain boundary conditions and initial conditions in figure (1-5). Dimensions and material of electrodes, conductivity of fluid, dimensions of channel and working chamber with inlet and outlet, gap between electrodes and position of magnets producing cross field make the difference in velocity of fluid. Fluid velocity found to be increasing when any of the field is increasing during experiments. These results in line with numerical results obtained . Bubble formation due to electrolysis has observed in the conducting fluid when operated with DC source which produces retardation effect. During investigations bubble formation got reduced when MHD system operated with AC source and fluid velocity enhances significantly. MHD system has been observed bidirectional capability on electric field reversal. Heating of fluid takes place because of electric current in conducting fluid which need to be investigated for control of temperature rise. Life of the MHD system can be significantly large as compared to system having moving parts, as there are no moving part in the proposed system

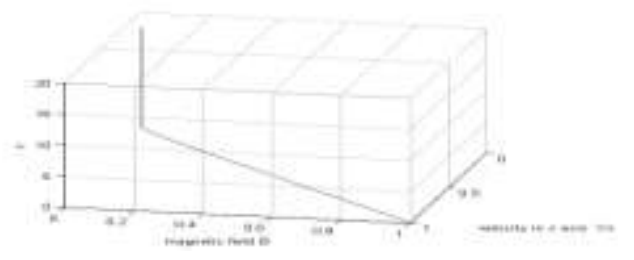

Figure 1 Velocity vx profile

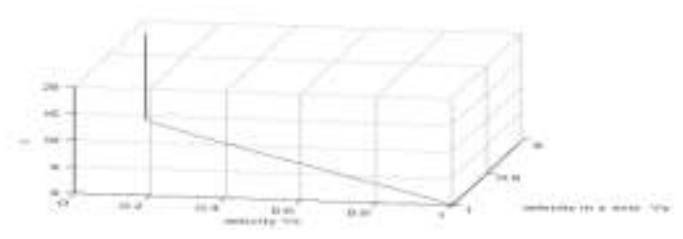

Figure 2 Velocity vy profile 


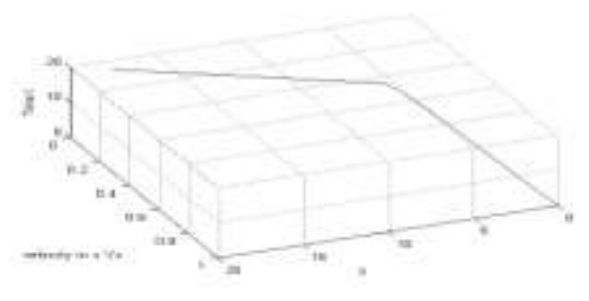

Figure3 Velocity vx profile

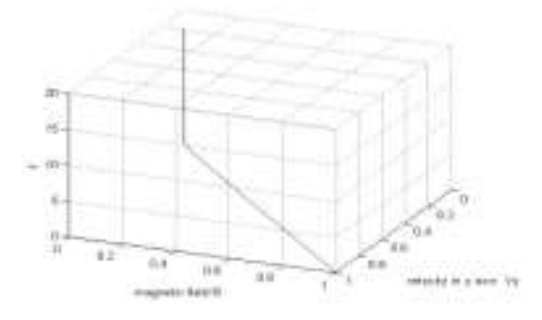

Figure 4 Velocity vy profile

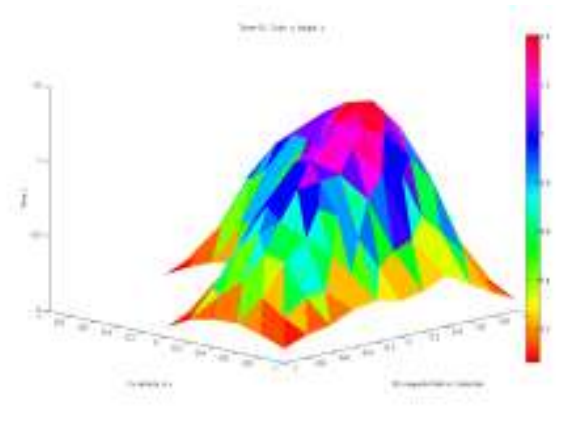

Figure 5 plot of $\mathrm{Vx}, \mathrm{Bz}$ and time $\mathrm{t}$

\section{PROTO-TYPE MHD SYSTEM}

Magneto hydrodynamic system resulting pumping action when an electromagnetic field interacts directly with the conducting fluid. The current flow in a direction perpendicular to the direction of magnetic field causes the fluid to experience a force. The force is in a direction perpendicular to both the magnetic field and the current flow. This high conductivity fluid can be used to produce a useful pumping effect. The measurement of bead velocity under the influence of MHD

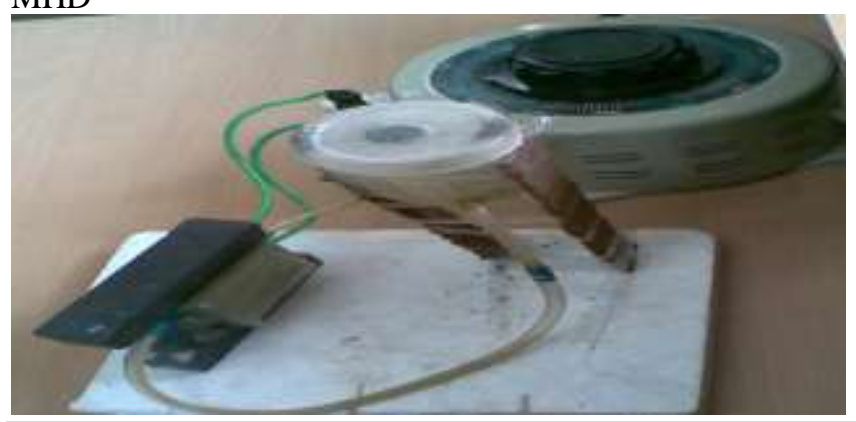

has been difficult due to strong electro kinetic flow in the sidechannel. We made visual estimations of bead velocity. Note that no movement in the main channel is observed when only the magnetic field is applied. We have fabricated MHD system using transparent cuboids having two electrodes of zinc plates of $7 \mathrm{cms}$ with inlet and out let ports and with an excitation connector for AC / DC operating voltage. We have designed and developed its proto type model and operated on DC and AC supply both. Experiment for flow of conducting fluid based on MHD principle have been realized, video for the same has been prepared. First we have operated our proto type MHD system with $12 \mathrm{~V}$ DC car batteries and then same system we tried to operate with $230 \mathrm{~V}$ AC supply with rheostat arrangement. We varied electric and magnetic fields one by one and noticed flow of fluid. It was found to be varying proportional to fields as effective result i.e. flow of conducting liquid found to be dependent on Intensity of the magnetic and electric field.

Figure 6 Prototype of MHD system developed

Direction of flow fluid also noticed to be varying depending on polarity of the supply. Direction of flow of fluid getting opposite on reversing its polarity. The MHD system used $\mathrm{NaCl}$ (salty water) as conducting fluid. Two electrodes of zinc strips were connected with $12 \mathrm{~V}, 20 \mathrm{Amp}$ hr battery later with $20 \mathrm{~V}-60 \mathrm{~V}$ ac supply with a on/off switch. Model was provided with provision of inlet and outlet pipe with two small tanks for storage of conducting fluid. We have operated $12 \mathrm{~V}$ DC switch and observed that flow of conducting fluid have taken place. Then with changed input dc supply with AC source varying from $20 \mathrm{~V}$ to $60 \mathrm{~V}$ and observed that there was significant enhancement in the velocity of the fluid as compared to DC source. These results were compared with analytical and numerical methods and found to be very close. More accurate results with data need better instrumentation for necessary set up and process to develop the same is under way.

An electrically conducting fluid $(\mathrm{NaCl})$ using Lorentz force. (Force produced when an electric current is applied across a channel filled with conducting solution in the presence of a perpendicular magnetic field). Flow measurements by mixing salt solution by recording a five seconds movie by mobile video capture camera. The measurements carried out by varying the $\mathrm{NaCl}$ concentrations and the experiments were carried out varying both, the magnetic field and electric field. Figure 1 -5presents numerical results and fig 6 has been for experimental test set up of proto type with inlet, outlet and MHD chamber with two permanent magnets.

\section{CONCLUSION}

To summarize, inspection of the flow velocity characteristics shows that the Lorenz force effect has successfully accelerated the electrolytic solution. From Lorenz force analysis, we noticed that the oscillation amplitudes of the velocity increase with the increasing of the current density and the magnetic flux density. So, the flow rate becomes more significant with the increase of magnetic and current density value. The obtained results confirm a directly influence of the 
external electromagnetic excitation and the chosen geometrical dimensions of the MHD. Bubble formation under DC source was observed inside the chamber. Bubbles produced in higher induced current not only retard flow motion, but also lower the hydraulic head.

This paper has presented for design and development with necessary simulation of MHD system. MHD system has been described by analytical, numerical and experimental methods. The concept has been new and has been worked for two dimensional solutions applying finite difference scheme. From results, we can conclude that the cited method achieves good convergence for velocity field and magnitude of flow rate can be more significant with AC source as compared with DC source, also formation of bubbles are less in AC source. Our research can be implemented for social usage in desert coolers as water sprinklers where we can implement pump without having any moving parts, with long life span as can be seen in our prototype model. We look forward for its realistic implementation. We have developed special case for $\mathrm{z}$ axis solution. Our future work shall be towards evolution of three dimensional MHD system taking Electric field into consideration. We have developed an efficient method for full system of PDE to reduced system of PDE. We intend to commercialize such MHD system in near future for social use and precision work in this direction is on the way.

\section{APPLICATIONS}

Microwave propulsion, Satellite propulsion, Space weather, MEMS Development, Micro fluidic devices, Sensors and Actuators, Precision Switch, Stirring of fluids and Desert Cooler water Sprinkler etc.

\section{ACKNOWLEDGEMENT \& BIOGRAPHY}

My Director Prof Asok De and Director NSIT Prof Raj Senani who inspired me for this research enriched me with all resources required. I am indebted of my wife Sujata, daughter Monica and son Rahul Yaduvanshi for giving the time and conducing environment to work towards this research.

Author: Rajveer S Yaduvanshi has 21 years of teaching and research work Author has successfully implemented fighter aircraft arresting barrier projects at select flying stations of Indian Air Force. He has worked on Indigenization projects of 3D radars at BEL as Senior Scientific Officer in Min of Defence. Currently he is working on MHD prototype implementation. He is teaching in ECE Deptt. of AIT, Govt. of Delhi-110031 as Asst Professor.

Co-Author: Prof. Harish Parthasarathy is an eminent academician and great researcher. He is professor and dean at NSIT. Govt. Engg. College at Dwarka, Delhi. He has extra ordinary research instinct and a great book writer in the field of Digital Signal Processing. He has published more than ten books and have produces more than seven PhDs in ECE Deptt. of NSIT,Delhi.

\section{REFERENCES}

Bahadir, A.R. and T. Abbasov (2005). A numerical investigation of the liquid flow velocity over aninfinity plate which is taking place in a magnetic field. International journal of applied electromagnetic and mechanics 21, 1-10.

[2] EM Lifshitz and LD Landau "Electrodynamics of continuous media "Butterworth-Heinemann.

[3] Fermigier, M. (1999). Hydrodynamique physique. Problèmes résolus avec rappels de cours, collectionsciences sup. physique, edition Dunod.

[4] Wait, R. (1979). The numerical solution of algebraic equations. A Wiley-interscience publication.

[5] Chorin, A.J., 1986, "Numerical solution of the Navier-Stokes equations" Math. Comp

Vol. 22, pp. 745-762.

[6] Cramer, K.R. \& Pai, S., 1973, "Magneto fluid Dynamics For engineers and Applied Physicists", McGraw-Hill Books.

[7] Feynman, R.P., Leighton, R.B. and Sands, M., 1964, "The Feynman Lectures on Physics" AddisonWesley, Reading, USA, Chapter 13-1.

[8] Gerbeau, J.F. \& Le Bris, C., 1997, "Existence of solution for a density dependant magnetohydrodynamic equation", Advances in

[9] Guermond, J.L. \& Shen, J., 2003, "Velocitycorrection projection methods for incompressible flows", SIAM J. Numerical Anal. Vol. 41, No. 1, pp. 112-134.

[10] Holman, J.P., 1990, "Heat Transfer" ( $7^{\text {th }}$ edition), Chapter 12, Special Topics in Heat Transfer, MacGraw-Hill Publishing Company, New York.

[11] PY Picard,"Some spherical solutions of ideal magnetohydrodynamic equations",Journal of non linear physics vol 14 no4 (2007),578-588. 